\section{Würdigung TARMED}

\author{
H. H. Brunner, Präsident FMH
}

Sehr geehrte Frau Kollega,

Sehr geehrter Herr Kollega

Die Verbindung der Schweizer Ärztinnen und Ärzte FMH führt zum zweiten Mal in ihrer Geschichte eine Urabstimmung unter ihren Mitgliedern durch. Sie ist der Einführung der sogenannten TARMED-Tarife gewidmet.

Wie Sie wissen werden, hat die FMH mit den Versicherern, vornehmlich der MTK (Medizinaltarifkommission der eidgenössischen Versicherer im Unfallversicherungsbereich UVG), der Invalidenversicherung IV und der Militärversicherung MV zuerst im sog. GRAT-Projekt, später in dessen Folgeprojekt TARMED (dort auch zusammen mit santésuisse [sas, vormals KSK], und $\mathrm{H}+$ als Organisation der Spitäler), diese Tarife entwickelt, die nun nach 11jähriger Arbeit eingeführt werden sollen.

\section{Allgemeine Bemerkungen}

Rufen wir uns noch einmal die wesentlichen Punkte in Erinnerung:

\section{Einheitliche Tarifnomenklatur}

Im Unfallversicherungsbereich UVG wie Krankenversicherungsbereich KVG wird nur noch eine Nomenklatur medizinischer Leistungen (Leistungsbezeichnung mit allfälliger Interpretation, Taxpunktzahlen) gelten. Dies stellt im KVG-Bereich eine grundsätzliche Neuerung dar, die aber vom KVG vorgegeben ist (Art. 43, Abs. 5 KVG).

Damit ist eine Zahl grundsätzlicher Implikationen verbunden:

- Ärztliche Leistungen können auch im KVG-Bereich hinsichtlich ausgelöster Kosten, Ort der Leistungserbringung und anderer Charakteristiken verglichen werden.

- Alle bisherigen Tarifstrukturen werden hinfällig und müssen durch die Tarifstruktur TARMED abgelöst werden, auf die sich der Bundesrat festgelegt hat. Entsprechend wurden alle bisherigen Tarifverträge gekündigt. Die Meinung, man könne bei Ablehnung der TARMED-Tarifstruktur mit den alten Tarifen weiterfahren oder eine andere Tarifstruktur anwenden, ist deshalb illusorisch.

- Taxpunktzahlen ergeben erst durch Multiplikation mit einem in Geldwert ausgedrückten Taxpunktwert den Betrag, mit dem ein Arzt* abge-

* Zur besseren Lesbarkeit des Textes werden nur männliche Formen verwendet. Wir bitten die Leserinnen um Verständnis. golten wird. Für den UVG-Bereich gilt schweizweit ein Taxpunktwert (TPW), der mit den Unfallversicherern auf Fr. 1.- festgelegt wurde. Im KVGBereich kommen prinzipiell mindestens 26 TPW (mindestens einer pro Kanton) zur Anwendung. Die entsprechenden Verhandlungen mit der santésuisse lagen und liegen in den Händen der kantonalen Gesellschaften; sie können erst beginnen, wenn über die Tarifstruktur bzw. die in einem Rahmenvertrag auf eidgenössischer Ebene niedergelegten Einführungsbestimmungen Konsens besteht. Die immer wieder gehörte Forderung, über TARMED sei erst abzustimmen, wenn auch die kantonalen TPW festgelegt seien, ist deshalb unerfüllbar.

\section{Anwendung der TARMED-Tarife}

Die TARMED-Tarife kommen nur im ambulanten Versorgungsbereich Arztpraxis und Spital ambulant, nicht aber im stationären Bereich zur Anwendung. $\mathrm{Zu}$ Beginn des GRAT/TARMED-Projektes war dies nicht klar; deshalb wurden eine umfassende Nomenklatur und Bewertung aller ärztlichen Leistungen (d.h. ambulant und stationär) durchgeführt. Dies hat sich, auch wenn eine Verrechnung ärztlicher Leistungen im stationären Bereich kaum direkt nach den TARMEDTarifen erfolgen wird, als zweckmässig herausgestellt, weil

1. die Abgrenzung ambulant vs. stationär erbrachter Leistungen fliessend ist, und

2. eine Überführung bzw. Anwendung in anderen Tarifsystemen (DRGs, andere Pauschalabgeltungen) damit möglich ist.

\section{Andere Medizinaltarife}

Neben den Arzttarifen existiert noch eine Vielzahl von Medizinaltarifen, die auch von Ärzten erbrachte Leistungen beinhalten. Zu nennen sind hier der Physiotherapietarif, der Zahnarzttarif und insbesondere die Analysenliste, die als eidgenössische Tarifstruktur für Laboranalysen angesprochen werden kann und die kantonalen Labortarife ablösen wird (soweit dies nicht bereits geschehen ist). Die arztrelevanten Leistungen aus diesen Tarifen wurden nicht in die TARMED-Tarife aufgenommen.

Für die Anwendung der erwähnten Nichtarzttarife gilt:

- Die Tarife werden auch durch Ärzte nach dem den Tarif begründenden Vertrag zwischen Leistungserbringern und Versicherern angewendet. Dies umfasst auch allfällige Sanktionsmassnahmen (Ausnahme Analysenliste).

- Die Analysenliste wird mit den entsprechenden Rahmenverträgen zum vom Eidg. Departement des Innern (EDI) festgesetzten TPW flächendeckend eingeführt. Allfällige Sanktionen werden im Rahmen der entsprechenden Rahmenverträge verhängt; die Aktivitäten von QUALAB in diesem Bereich finden mit Wirksamwerden der Rahmenverträge ihr Ende. 


\section{Geltungsbereich}

Die TARMED-Tarife werden formal nur im Geltungsbereich der sozialen Krankenversicherung, der Unfall-, Invaliden- und Militärversicherung angewendet, nicht aber im Zusatzversicherungsbereich. Dieser bei einer immer grösseren Zahl anbietender Ärzte stetig schrumpfende Zusatzversicherungsbereich ist unter dem Druck der Wettbewerbskommission (WEK0) zunehmend dem freien Markt bzw. dem Druck auf Preissenkungen ausgesetzt; tarifarische Regelungen wurden ausser Kraft gesetzt. Blauäugig wäre es aber, den TARMED-Tarifen die Wirkung einer Messlatte abzusprechen. Diese Vorgänge sind der Hintergrund für die Opposition operativ bzw. interventionell tätiger Ärzte.

\section{Einkommenseinbussen?}

- Ein Vergleich der Preise häufig erbrachter, ambulanter Leistungen nach aktuellen Sozialversicherungstarifen mit den TARMED-Tarifen zeigen mengengewichtet für einen TPW Fr. 1.- keine signifikanten, d. h. $<10 \%$ betragenden Einkommenseinbussen, wenn die neuen Tarife korrekt angewendet und das Augenmerk nicht ausschliesslich auf den Preis der Leistung im engeren Sinne gerichtet wird. Einzige Ausnahme bleiben die Radiologen, zu deren Existenzsicherung bis zur Einführung der Tarife etwas geschehen muss; sollte dies nicht möglich sein, so wird der Zentralvorstand auch bei positivem Entscheid der Urabstimmung die Einführung der Tarife blockieren.

- Preis- bzw. Einkommensvergleiche werden dadurch erschwert, dass sich in der Anwendung der meisten Tarife Eigenheiten eingeschlichen haben, die hinter dem Buchstaben der Tarife eine eigene tarifferne Abgeltungsrealität entstehen liessen. Dies trifft für die meisten Tarife zu, sei es Spitalleistungskatalog, seien es kantonale KVG-Tarife. Nur die von NewIndex angestellten Vergleichsberechnungen aufgrund einer die Verrechnungsrealitäten materiell richtig abbildenden Transcodierung liefern brauchbare Resultate. Vergleiche ausschliesslich auf der Basis buchstabengetreuer Anwendung der aktuellen Tarife sind deshalb, wer auch immer sie angestellt hat, unsinnig und irreführend.

\section{Daten/Datenschutz}

Bestreben der Krankenversicherer war und ist es, die Einführung der TARMED-Tarife zum Anlass zu nehmen, in den Besitz möglichst detaillierter Patientenund mehr noch Leistungserbringerdaten zu gelangen. Es handelt sich hier ethisch, juristisch und politisch um einen ausserordentlich heiklen Bereich. Grundlinien der unserer Politik verletzenden Zugeständnisse sind in diesem Bereiche unvorstellbar.

- Vorab sind es Regelungen des Datenschutzes, die hier Grenzen setzen. Leider war es nicht möglich, diese Fragen mit den Funktionären des Datenschutzes in allen Aspekten zu klären.

- Die Krankenversicherer verlangen eine Offenlegung aller Mitgliederdatenbanken der FMH nach dem sog. Bringprinzip. Konkret würde dies bedeuten, dass alle Kenndaten unserer Mitglieder den Versicherern, d.h. jeder einzelnen Kasse und ihren Geschäftsstellen, regelmässig auf elektronischem Datenträger zur Verfügung gestellt werden müssten, und dies noch ohne jede glaubhafte Gewähr auf Geheimhaltung. Das Verhalten der Krankenversicherer ist, aus ihrer Sicht gesehen, durchaus verständlich: Sie wollen gleichsam «avant la lettre" in den Besitz dieser Daten kommen, um nach Aufhebung des Kontrahierungszwanges die ihnen genehmen Ärzte auslesen zu können. Da es offensichtlich doch nicht so einfach ist, entsprechende Daten selber zu generieren und zu verwalten - SWICA lässt grüssen -, ist es viel einfacher, die benötigten Daten per Vertrag und erst noch zum Nulltarif bei dem zu besorgen, der über die Daten ist bester Qualität verfügt.

Solches ist selbstverständlich undenkbar und Grund dafür, dass der entsprechende Anhang im Rahmenvertrag mit den Krankenversicherern nicht vorliegt bzw. der Abstimmung nicht unterbreitet werden kann. Sollte es doch noch zu einer vertraglichen Regelung mit der santésuisse kommen, so würde diese der Ärztekammer zum Beschluss unterbreitet werden. Vor einem Beschluss durch die Ärztekammer wird der Zentralvorstand auch bei positivem Urabstimmungsergebnis die Einführung der TARMED-Tarife blockieren.

An den Versicherern ist es nun, endlich zu begreifen, dass Daten- und Persönlichkeitsschutz nicht Verhandlungsmasse sind, über die man sich mit steinzeitlichen Drohgebärden hinwegsetzen kann.

\section{Dignitätskonzept}

Das sog. Dignitätskonzept wie auch das Spartenkonzept sind zur Handhabung der Tarifstruktur unerlässliche Instrumente, die als solche der Tarifstruktur zuzuordnen und deshalb im Rahmen der Urabstimmung in der von der Projektleitung TARMED erwahrten Version 9.0 (Dignitätskonzept) bzw. Version 28.8.01 (Spartenkonzept) mit der Tarifstruktur zusammen dem Beschluss durch die Ärzteschaft unterworfen sind. Die Konzepte sind aber nicht Teile der Tarifstruktur gemäss Art. 43, Abs. 5 KVG, und unterliegen deshalb auch keinen Interventionen seitens der Behörden.

Zusammengefasst setzen die Regelungen des Dignitätskonzepts fest, welche Leistungen Ärzte gemäss ihrer Weiterbildung abrechnen dürfen; dies wird in der Tarifstruktur unter der Rubrik qualitative Dignität rubriziert. Die sog. quantitative Dignität ist für den einzelnen Anwender der TARMED-Tarifstruktur nicht massgeblich; sie gibt nur an, nach welcher Dignität die entsprechende Leistung kalkuliert wurde. Die Angaben stehen für jede Leistung in der Closed-User-Group (CUG) unter www.tarmed.ch zur Verfügung; im Tarifbrowser wurde sie nicht aufgeführt, weil der Unterschied der Begriffe quantitative und qualitative Dignität offensichtlich schwer verständlich und verwirrlich ist. 


\section{Reegineering}

Die Version 1.1. der TARMED-Tarifstruktur bedarf vor ihrer Einführung noch einer grundsätzlichen Überprüfung der Eck- und Steuerwerte, der Verknüpfung derselben bzw. des Kalkulationsalgorithmus sowie der zugrunde gelegten Daten. Dies als Reengineering II (RE II) bezeichnete Vorhaben muss vor Einführung im KVG-Bereich, d.h. vor dem 31. Dezember 2002, abgeschlossen sein. Sollte dies nicht erreicht werden können, so würde der Zentralvorstand die Einführung der TARMED-Tarife blockieren.

Dies gibt zu einer grundsätzlichen Anmerkung Anlass: Eine Tarifstruktur kann nie "definitiv" sein, es sei denn, jegliche Entwicklung in der Medizin käme zum Stillstand. Zustimmung kann deshalb immer nur Zustimmung zu momentan Erreichtem sein.

Obwohl finanzielle wie operative Gründe dafür gesprochen hätten, Urabstimmung und Dignitätserhebung miteinander durchzuführen, hat der ZV, auch nach einer Konsultativabstimmung in der Ärztekammer, darauf verzichtet, dies zu tun. Massgeblich war der Wille, jeglichen Eindruck einer Einflussnahme auf die laufende Urabstimmung zu vermeiden.

\section{Aktuelle Kritiken}

1. TARMED enthält immer noch eine Unzahl von Fehlern und ist so nicht akzeptabel

- Vorab ist ohne Wenn und Aber zuzugestehen, dass es auch bei den Umsetzungsarbeiten zu Version $1.1 \mathrm{zu}$ materiell relevanten Fehlern gekommen ist. (Nicht beschlusskonforme Umsetzung Version 1.0 zu Version 1.1; Psychiatrie: Fehler Rückführung Faktor 1,2; Delegierte Psychotherapie: nicht nur für psychiatrische Fachärzte; XML-Standard: nicht in Tarifstruktur zu verankern).

- Vorwürfe solcher Art wurden nicht zuletzt in der Romandie erhoben; verantwortlich sind hier in der Regel Probleme bei der Übersetzung vom Deutschen ins Französische.

- Als Fehler wird aber sehr häufig der Umstand bezeichnet, dass eine ärztliche Forderung nicht verwirklicht werden konnte. Untergründig ist hierfür eine falsche Vorstellung von Tarifverhandlungen massgeblich: Es ist keineswegs so, dass die Ärztedelegationen ihre Forderungen einer Rezeptur gleich einbringen können; im Gegenteil ist es so, dass seitens der Versicherer in der Regel alle Forderungen bestritten bzw. mit einer Gegenforderung beantwortet werden.

Man kann eine solche Form von Verhandlungen als falsch und unzweckmässig beklagen; sie ist in der Schweiz (wie übrigens den meisten anderen Ländern auch) gesetzlich abgesicherte oder gar vorgeschriebene Realität.
2. TARMED enthält mehr als 2000 den Patienten gefährdende und die Qualität der ärztlichen Versorgung gefährdende Leistungen

Diese Vorwürfe müssen als unqualifiziert und demagogisch bezeichnet werden:

- Der Einwand wird erst jetzt gemacht, obwohl sich die Nomenklatur seit ihrer Version 2.2 nicht mehr grundlegend verändert hat. Und dies durch Exponenten von Fachgesellschaften, die seit Beginn und bis in die Jetztzeit an der Erarbeitung der Tarifstrukturen nachweisbar mitgearbeitet und ebenso nachweisbar diese Einwände nicht gemacht haben.

- Im internationalen Preis-Leistungs-Vergleich liegen die TARMED-Tarife an der Spitze. In den Ländern mit zum Teil weit tieferer Preis-LeistungsRelation konnten tarifinduzierte Erhöhungen von Morbidität und Mortalität nicht festgestellt werden.

- Die Exponenten dieser Kritik konnten bis jetzt keine einzige Leistung namhaft machen, die die im Kern ungeheuerliche Unterstellung rechtfertigen würde. An der Ärztekammer wurden drei Beispiele zitiert, die nichts über die insinuierte Patientengefährdung, mehr aber über fehlendes Tarifverständnis der Zitierenden Zeugnis ablegten.

3. Die Einführung von TARMED kostet jede Praxis Tausende von Franken für die Aufrüstung der Praxis-/Abrechnungssoftware

Dies ist ein Mythos, der von Softwareanbietern verbreitet wird, um sich, die Gunst der Stunde nutzend, ein kleines (oder besser grosses) Häppchen abzuschneiden bzw. allenfalls lang anstehende UpgradingMassnahmen zu vergolden: len,

In der Sache geht es darum, ein System zu erstel-

- das TARMED-tauglich ist und Taxpunkte AL und TL zu verschiedenen TPW zusammenzählen kann;

- das dem Arzt gemäss qualitativer Dignität zustehende Leistungen zur Verrechnung zulässt;

- das Medikamente, Verbandsstoff usw. (wie bisher) und Tarife Physiotherapie, SSO und Analysenliste enthält.

Die Unterlagen hierfür sind allen Softwarehäusern seit mehr als einem Jahr spätestens bekannt (und auch in der CUG TARMED verfügbar). Das Problem liegt an einem ganz anderen Ort:

- Viele Softwarehäuser wollten nicht investieren, solange nicht definitiv über die Einführung von TARMED befunden sei.

- Diese Anbieter haben nun Mühe, die Software all ihrer Kunden bis zu einem Einführungstermin spätestens 1. Januar 2003 aufzurüsten. Die Kosten hierfür können, solange sie etwas mit Aufwand $\mathrm{zu}$ tun haben, entsprechend den obigen Ausführungen nicht in Tausende von Franken gehen. 
4. Über TARMED kann nicht befunden werden, solange nicht alle kantonalen Taxpunktwerte bekannt sind

Diese Position ist prima vista absolut verständlich; niemand kauft Bananen mit einer Taxpunktzahl als Aufschrift, die erst an der Kasse nach einem unbekannten Taxpunktwert zum definitiven Preis ausmultipliziert wird. Es ist die einmal mehr dem «common sense» nicht zugängliche Gesetzeslogik, die dies verlangt:

- Für den $U V / M V / I V$-Bereich ist dies möglich, weil es sich um einen eidgenössischen Tarif mit EINEM TPW handelt, der zwischen den Vertragsparteien - FMH und eidgenössische Sozialversicherer abschliessend ausgehandelt werden kann. Wie den Unterlagen entnommen werden kann, wurde dieser TPW, vorbehältlich der gesetzlich vorgeschriebenen Zustimmung durch den Bundesrat, mit Fr. 1.- ausgehandelt.

- Grundsätzlich anders präsentiert sich die Situation im KVG-Bereich: Hier muss in 26 Kantonen der TPW ausgehandelt, durch den Preisüberwacher abgesegnet und die kantonale Regierung genehmigt werden, basierend auf einer Tarifstruktur, die auf eidgenössischer Ebene festgelegt wird.

- Diese Topologie macht klar, dass TPW-Verhandlungen in den Kantonen erst dann beginnen können, wenn diese Tarifstruktur auf eidgenössischer Ebene festgelegt ist. Die Ausführungen zeigen, dass die Forderung nach gleichzeitiger Abstimmung über Tarifstruktur UND kantonale TPW/KVG verhandlungstechnisch bzw. aus gesetzlichen Gründen unerfüllbar ist.

\section{Ausblick}

In den letzten Wochen ist in der Öffentlichkeit aber auch in der Ärzteschaft der Eindruck entstanden, die nun anlaufende Urabstimmung werde über Wohl oder Weh der TARMED-Tarife abschliessend entscheiden. Dies trifft nicht zu, vor allem für den Fall eines JA:

- Nachdem die FMH sich bis jetzt nicht (wie von vielen insgeheim erhofft) als TARMED-Killer erwiesen hat, scheinen andere Vertragsparteien in die Bredouille zu geraten: Die santésuisse hat dem Rahmenvertrag bis jetzt nicht zugestimmt, die letzte Stellungnahme von $\mathrm{H}+$ stellt mehr Fragen als sie Antworten gibt.

- Alle Beschlüsse der Vertragsparteien wie auch der zuständigen Behörden unterliegen der Klagemöglichkeit. Es kann mit Sicherheit davon ausgegangen werden, dass hiervon Gebrauch gemacht werden wird, umso mehr als die Rechtswege lang und mit Zeitgewinn verbunden sind (vgl. Zemp Gsponer C, Bitzi F. TarMed und Art. 6 EMRK. Besteht ein Anspruch auf gerichtliche Überprüfung nach der Europäischen Menschenrechtskonvention? Schweiz Ärztezeitung 2001;82[18]:924-7). Seitens der zuständigen Behörden wird das Problem heruntergespielt und banalisiert; konkrete Massnahmen wurden von dieser Seite nicht getroffen.
Dies kann und soll aber nicht Anlass für die FMH sein, ihre Entscheide zu extemporieren, im Gegenteil. Im Zusammenhang mit diesen Entscheiden wird immer wieder die Frage nach den Konsequenzen einer Ablehnung der TARMED-Tarife durch die FMH aufgeworfen. Vorab ist festzuhalten, dass in keinem Land der Welt - und Beispiele gibt es - ein Arztleistungstarif gegen den erklärten Willen der Ärzteschaft eingeführt werden konnte. Dies würde wohl auch in der Schweiz für den Fall zutreffen, dass die politischen Behörden, z.B. mittels eines Dringlichen Bundesbeschlusses (DBB), die Tarifstruktur TARMED Version 1.1 einführen und die Anwendung, insbesondere den TPW, mittels einer Verfügung festlegen würden.

Die Konsequenzen eines solchen Schrittes wären absehbar:

- Die Ärzteschaft bzw. die FMH würden jegliche Kooperation für die Anwendung der Tarife verweigern und die tarifarischen Regelungen unterlaufen. Der Tarif würde damit in grossen Teilen unanwendbar.

- Zwischen Versicherern und politischen Instanzen einerseits, der Ärzteschaft andererseits würde ein chronischer Kriegszustand herrschen. Ärzte würden etwas tun, was sie bis jetzt nicht getan haben: Diese Institutionen in grosser Geschlossenheit frontal angreifen. Wahrlich kein Vergnügen für Institutionen, die schon jetzt mit erheblichen Glaubwürdigkeits- und Imageproblemen $\mathrm{zu}$ kämpfen haben.

Dem gegenüber stehen aber vor allem langfristig wirksame negative Konsequenzen:

- Medien wie Politiker und Versicherer erhielten einmal mehr die Gelegenheit, die Ärzteschaft als die ewigen, für alle Übel des Gesundheitswesens verantwortlichen Betonierer abzustrafen und so von der eigenen Unfähigkeit und Konzeptlosigkeit abzulenken.

- Die Lust vornehmlich vieler Politiker, diese Ärzteschaft nun mit allen gesetzlichen-juristischen Mitteln botmässig zu machen, würde steigen und die Entwicklung eines befriedigenden Berufsumfeldes unter der Leitidee Autonomie ärztlicher Tätigkeit verhindern.

- Die Ärzteschaft wäre "weg vom Fenster»; alle weiteren Entscheide könnte sie nicht mehr konstruktiv mitgestalten.

Im Kern reduziert sich der anstehende Entscheid auf eine relativ einfache Frage:

- Gibt die Ärzteschaft einem zweifelsohne nicht vollkommenen und noch weiter entwicklungsbedürftigen Tarifwerk ihre Zustimmung und hält sich ihre Kräfte für weit wichtigere Entwicklungen und Auseinandersetzungen frei, oder

- ist ihr der Kampf um ein stimmiges Tarifwerk so wichtig, dass sie hierfür Geld und insbesondere strategische Positionen opfert? 
Um diesen Kernentscheid dreht sich die Urabstimmung, die in diesem Sinne völlig offen und nicht ein vom Zentralvorstand der Ärztekammer vorgespurtes Ja ist.

In dieser sehr schwierigen Situation zeichnen sich bei vielen Mitgliedern drei Reaktionen ab:

1. Wir verrechnen nach unserem eigenen Tarif, z.B. nach einem FMS-Tarif

Diese Idee ist ganz einfach unrealisierbar:

- Tarifssysteme müssen in der Schweiz mit den Versicherern ausgehandelt und durch die zuständigen staatlichen Behörden abgesegnet werden. Einseitige Akte sind aus rechtlicher Sicht prinzipiell undenkbar (was nicht bedeutet, dass die FMH nicht eine Tarifstruktur TARMED korrekt erarbeiten kann [und wird!], die die Fehler der jetzigen Tarifstruktur aufzeigt).

- Dieser generelle Grundsatz wird dadurch verdeutlicht, dass gemäss dem schon zitierten Art. 43, Abs. $5 \mathrm{KVG}$, in der Schweiz nur eine Tarifstruktur für die Verrechnung von Einzelleistungen angewendet werden kann.

2. Entscheid weiter hinausschieben, bis Tarifstruktur besser, alle TPW bekannt, Einführungszeitpunkt genau bekannt sind

Die Gremien haben erkannt, dass diese Strategie, so es denn eine wäre, ins Aus führen müsste:

- Der Entscheid muss jetzt fallen; jedes Hinauszögern wird als Nein interpretiert.

- Verbesserungen der Tarifstruktur, vor allem im Bereiche des Rechnungsmodells, sind möglich und werden von den anderen Parteien akzeptiert werden (vgl. Punkt 7). Sie sind aber nur zu erreichen, wenn die FMH dem aktuellen Verhandlungsergebnis nun zustimmt und damit am Verhandlungstisch bleibt.

- Und noch einmal: Die Hoffnung, mit den alten Tarifen und damit der alten Ordnung fortfahren zu können, ist illusorisch, weil nicht gesetzeskonform.
3. TARMED ist (nicht mehr) unsere Sache; "Frau Dreifuss" - als Summa des Politischen verstanden - soll den TARMED-Tarif einführen und damit auch die gesamte Verantwortung übernehmen

Diese Position ist ebenso verführerisch wie suizidal:

- Sie kann kurzfristig zu Zeitgewinn und Entlastung führen.

- Sie wird aber nie und nimmer die Einführung von TARMED oder anderer darauf aufbauender $A b$ geltungssysteme verhindern können .... und dies dann zum TPW à la mode de Dreifuss.

- Und das Fatale: Dieses Vorgehen wäre das Eingeständnis der FMH, dass die Ärzteschaft ureigenste Geschäfte nicht mehr selber regeln kann und damit die Autonomie des ärztlichen Berufes und seiner Berufsorganisation der Vergangenheit angehören. Oder in lateinischer Prägnanz: Finis Foederationis Medicorum Helveticorum FMH.

Wer sich aus Schwäche die Vögte ins eigene Land holt, wird sich nicht wundern dürfen, wenn sich diese Vögte dann in diesem Land auf Dauer häuslich einrichten. Dies ist im Positiven wie Negativen 700jährige schweizerische Erfahrung, die auch heute, von Romanshorn bis Genf, noch ihre Gültigkeit hat.

Zentralvorstand wie Ärztekammer empfehlen Ihnen ein dreifaches JA mit Zustimmung zur Tarifstruktur sowie zu den Rahmenverträgen für den Bereich UV/IV/MV wie auch den Bereich KVG. 